\title{
Rolling System Design Using Evolutionary Sequential Process Optimization
}

\author{
Ashutosh Tiwari, Victor Oduguwa, and Rajkumar Roy
}

\begin{abstract}
The design of a rolling system is a multistage process optimization problem involving sequential relationship between consecutive stages. This relationship is peculiar to sequential processes in which the output stock of one stage serves as the input stock into the deforming tool of the other stage. This paper describes the optimization of a real-life rolling system design using a genetic algorithm (GA)-based approach capable of dealing with the sequential nature of this problem. It presents a mathematical model of a real-life rolling system design and explains the proposed optimization approach. Even in the presence of multiple stages, the proposed approach identifies a variety of near-optimal design solutions from which one could be finally chosen based on designer's preferences. It is also shown that the obtained solutions dominate the designs reported in literature.
\end{abstract}

Index Terms-Genetic algorithms (GAs), multiobjective optimization, multistage process optimization, rolling system design (RSD), sequential process optimization (SPO).

\section{INTRODUCTION}

$\mathbf{T}$ HERE IS AN ever-increasing demand in the process industry to become more flexible, responsive, and energy efficient. The competition is fierce; complex products are being required at higher quality for the same cost with margins continuously being squeezed. In order to succeed in such a competitive environment, the industry is seeking innovative intelligent solutions to optimize its processes. Process optimization involves the generation of optimal design solutions for individual units of the process including both design information (such as the geometrical size of a unit) and the operating conditions for the unit. Process optimization problems are complex and can be characterized by their multiple stages and hierarchical nature. Individual units of process systems are connected to form multiple stages of the overall process problem. The nature of this association could be sequential (as seen in metal forming processes) or nonsequential (as seen in network optimization problems). The sequential nature of the process offers the separate subsystems a dependency link where the output of a subsystem becomes the input of the subsequent subsystem in an orderly manner. Nonsequential is nonordered association. This paper focuses on rolling system design, which is a sequential process optimization problem.

A sequential process optimization (SPO) problem is a nested problem in which decision-making is sequential from higher to lower level, characterized by decisions made at one level in-

Manuscript received March 30, 2006; revised December 29, 2006 and January 23,2007 . This work was supported in part by the Engineering and Physical Sciences Research Council (EPSRC) and in part by Corus U.K.

The authors are with the Decision Engineering Centre, Cranfield University, Cranfield, Bedford MK43 0AL, U.K. (e-mail: a.tiwari@ cranfield.ac.uk; voduguwa@hotmail.com; r.roy@cranfield.ac.uk).

Digital Object Identifier 10.1109/TEVC.2007.896688

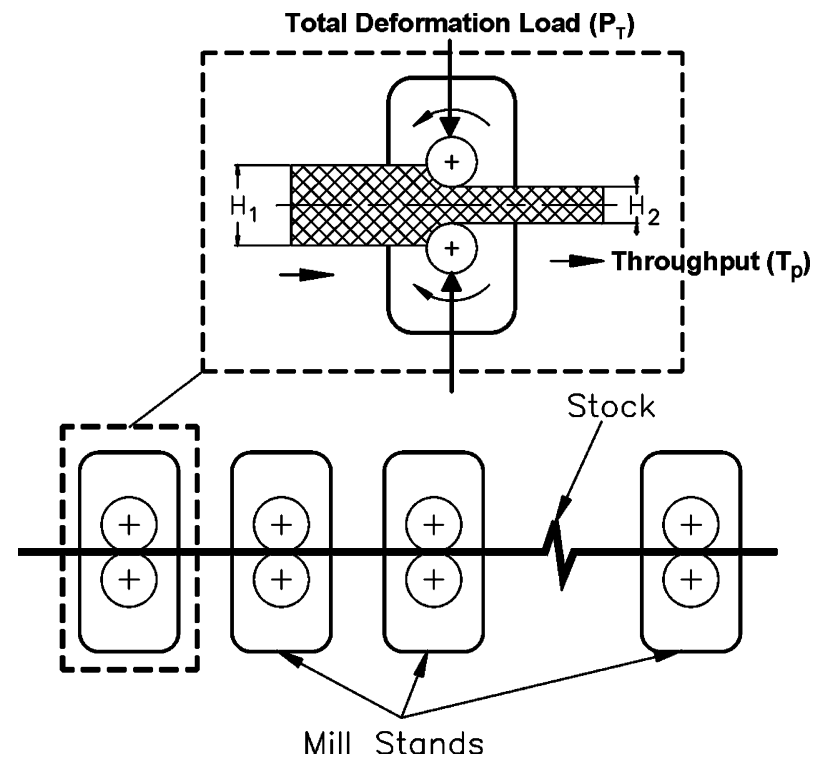

Fig. 1. A multipass rolling system.

fluencing the decisions made at a lower level. The behavior of the subsequent subsystem is influenced by the behavior of the previous subsystem [1]. Thus, an important feature of the SPO problem is that the objective functions of each unit may be partially determined by variables controlled by other units operating at other levels. Real-world SPO problems are usually large scale and complex [17]. The design of a rolling system is a multiobjective, multistage SPO problem involving a large number of design variables and a sequential relationship between consecutive stages. This paper describes the optimization of a real-life rolling system design using an approach based on genetic algorithms (GAs).

\section{Modeling a Multipass Rolling SYSTEM DESIGN (RSD)}

The multipass rolling system design (RSD) attempts to locate optimal design solutions for individual passes of the rolling process including both design information (such as the geometrical size of a roll) and the operating conditions for the mills. As shown in Fig. 1, the multipass rolling system is a high-speed continuous metal forming process where the metal from the reheating furnace (known as stock) is continuously deformed into the desired geometry by passing through a series of rotating cylindrical rolls.

\section{A. General Mathematical Formulation of SPO Problems}

The mathematical formulation of the SPO problem is presented in (1) and (2), and illustrated in Fig. 2. Equation (1) shows 


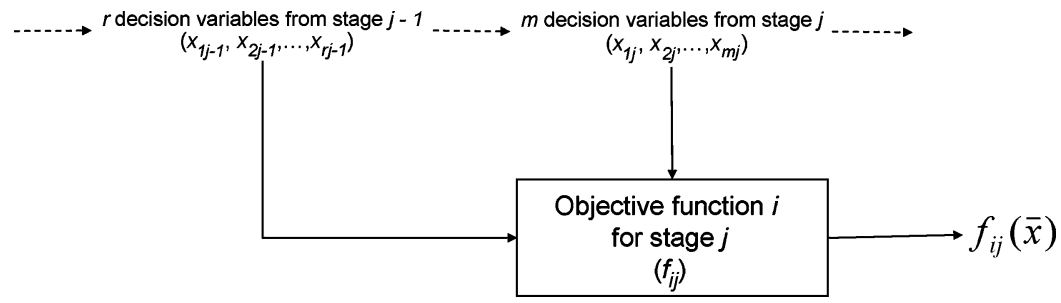

Fig. 2. Mathematical formulation of the SPO problem.

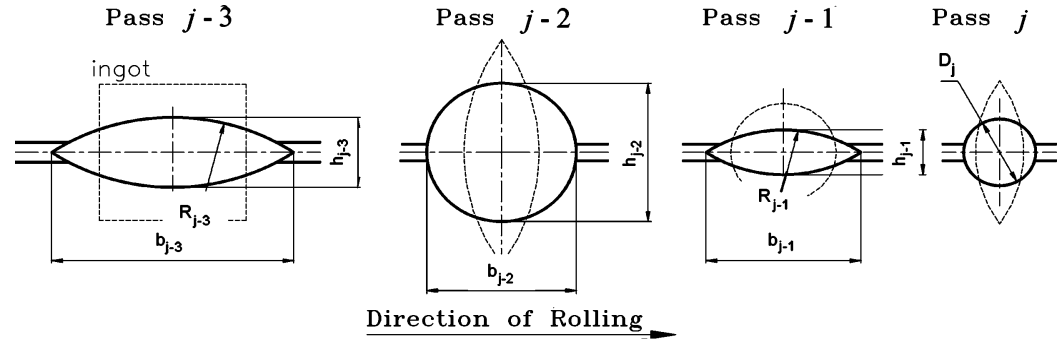

Fig. 3. Round-oval-round breakdown sequence.

the objective function $i$ for an individual stage $j$, and its functional relationship with $m$ decision variables from stage $j$ and $r$ decision variables from stage $j-1$. The decision made at stage $j$ is influenced by the $r$ decision variables from stage $j-1$. This represents how multistage dependencies are represented in the mathematical formulation of SPO problems. Only a subset of variables from stage $j-1$ influence the decision made at stage $j$. This explains the condition $r<m$ in (1). Equation (2) gives the objective function for the overall system. Here, $i$ is the $i$ th objective, $p$ is the number of objectives, and $n$ is the number of stages. This equation depicts that the overall objective function is dependent on the values of objective functions from individual stages. $m$ and $r$ are considered to be constant for all stages. Most real-life SPO problems satisfy this condition. However, the mathematical formulation presented here can easily be generalized by using $m_{j}$ and $r_{j}$ as $m$ and $r$ values at stage $j$

$$
\begin{aligned}
f_{i j}(\vec{x})= & \left\{f_{i j}\left(x_{1 j}, x_{2 j}, \ldots x_{m j}, x_{1 j-1}, x_{2 j-1}, \ldots x_{r j-1}\right)\right. \\
& \mid(j=1,2, \ldots n), r<m\} \\
F_{i}(\vec{x})= & \left\{F\left(f_{i 1}(\vec{x}), f_{i 2}(\vec{x}), \ldots, f_{i n}(\vec{x})\right) \mid i=1,2, \ldots p\right\} .
\end{aligned}
$$

\section{B. Development of Multipass Rolling Model}

This section develops the mathematical model of a RSD using the generic formulation in Section II-A. Fig. 3 shows the process geometry. The dashed line shows predeformation profile and the solid line shows postdeformation profile at each stage. $b$ is the stock width, $h$ is the stock height, $R$ is the radius of curvature, and $D$ is the diameter. The overall breakdown sequence consists of a number of cascaded passes. The $j$ th pass is denoted by $P_{j}$. Each pass is physically separated from its neighbors, and the output from the $P_{j-1}$ is provided to the $P_{j}$ as input. The breakdown sequence shows the oval stock turned through $90^{\circ}$ with its major axis in-line with the vertical axis of the round pass. This is repeated for all the subsequent passes. A detailed description of the model is available by Oduguwa [11].

\section{1) Objective Functions:}

Throughput: Throughput $\left(T_{p}\right)$ is an important roll design objective that expresses the effect of mill productivity. It is a suitable measurement of how well the current schedule is meeting the requirement. It is expressed in terms of exit speed $(w)$ and final stock area $\left(A_{2}\right)$

$$
T_{p}=A_{2} w .
$$

Roll force: One of the most important objectives in scheduling is to provide an optimum rolling load $\left(P_{T}\right)$ required for deformation at each stand. Excessive loading in various passes can affect the productivity of the rolling process. The total deformation load cost function for total $N_{p}$ number of passes is defined as

$$
P_{T}=\sum_{j=1}^{N_{p}}\left(P_{j}\right)
$$

where $P_{j}$ is the roll load at pass $j$. Shinokura and Takai [16] proposed a simple equation for calculating the nondimensional roll force and torque arm coefficient expressed as a function of the geometry of the deformation zone. This formulation is adopted in this study since it is suitable for oval-to-oval passes.

2) Constraints: The total roll load is limited to the mechanical design limits of the rolling mills imposed by the roll manufacturers. Excessive roll load can cause roll breakage that can be detrimental to production efficiency. This constraint can be formulated as follows:

$$
g_{1}(x) \equiv P_{j} \leq P_{\max }
$$

where $P_{j}$ is the roll load at pass $j$.

$\lambda_{T}$ and $\lambda_{j}$ are the coefficients for total elongation and elongation at stage $j$, respectively, therefore

$$
g_{2}(x) \equiv \sum_{j=1}^{n} \lambda_{j}-\lambda_{T} \geq 0 .
$$


The following constraint is required to ensure that the breakdown sequence is achieved with reduction taking place from pass to pass. For every pass $j$

$$
\text { Interpass reduction } \quad g_{3}(\mathbf{x}) \equiv \lambda_{j}>1
$$

where $\lambda_{j}$ is the coefficient of elongation for $j$ th pass.

The overall rod size is a measure of product quality. This is allowed to vary within a given tolerance limit $K . K$ is treated as a soft constraint to allow the possibility to explore designs within the specified boundaries. This is specified as

$$
g_{4}(x) \equiv K_{\min } \leq \sqrt{\left(D_{N p}-D\right)^{2}} \leq K_{\max }
$$

where $D$ is the roll diameter and $N_{p}$ is the total number of passes (Fig. 3).

The breakdown sequence (Fig. 3) shows the oval stock turned through $90^{\circ}$ with its major axis in the vertical diagonal of the square pass. The square stock is provided as flat into the oval pass and the oval stock is turned through $90^{\circ}$ with its major axis in-line with the vertical axis of the round pass. Constraints on interpass sections can be shown as

$$
\begin{aligned}
& g_{5}(\mathbf{x}) \equiv b_{j}^{\mathrm{var}}=h_{j-1}^{\mathrm{var}+1} ; \quad j=1,2 \ldots n ; \operatorname{var}=1,2 \\
& g_{6}(\mathbf{x}) \equiv h_{j}^{\mathrm{var}}=b_{j-1}^{\mathrm{var}+1} ; \quad j=1,2 \ldots n ; \operatorname{var}=1,2
\end{aligned}
$$

where $j$ is the pass number, var $=(1,2)$ for inlet and outlet variables, respectively, $n$ is the final pass number, $b$ is the stock width, and $h$ is the stock height.

3) Complete Mathematical Formulation: A formal definition of the multiobjective RSD optimization problem is presented below

$$
\begin{aligned}
& \text { Minimize Total Deformation Load (4) } \quad f_{1}(\mathbf{x}) \equiv P_{T}(x) \\
& \text { Maximize Throughput (3) } \quad f_{2}(\mathbf{x}) \equiv T_{p}(x) \\
& \text { Subject to : } \text { Roll load (5), Total elongation (6), } \\
& \text { Interpass reduction (7), Rod size (8), and } \\
& \text { Interpass section constraints (9) and (10). }
\end{aligned}
$$

The aim is to minimize deformation load and maximize rolling throughput, subject to given constraints for a four-pass oval to round design. The deformation load is a cost objective, while throughput is a function of mill productivity. Excessive deformation load results in excessive roll wear and, hence, overall production cost. Both objectives are assumed to be conflicting in nature since metal compression by the cylindrical rolls encourages metal flow in the direction of rolling, which also increases the metal deformation load. Fig. 1 illustrates the conflict between the two objectives of this problem.

\section{EXISTING APPROACHES}

A review of literature reveals that there is very little work reported on optimization approaches for dealing with SPO problems. Classical methods of solving these problems are based on trial and error using mainly empirical guidelines, supported by experience [13]. The optimization task, therefore, becomes slow and often results in suboptimal solutions. Kobayashi [9] applied a finite-element (FE)-based backward tracing technique to design a preform in a shell housing. This technique tends to be more efficient when the loading path is known; however, this can lead to difficulties in those problems in which the search space is unknown, especially when multiple diverse loading paths are present. Dynamic programming can handle continuous and discrete variables; however, it is limited in its use since the SPO problems normally involve a large number of process variables with a wide range of values, which creates problems for dynamic programming. Some authors have used derivative-based techniques and direct search methods for solving SPO problems [7]. However, the objective functions and constraints are required to be twice differentiable creating difficulties for SPO that generally reveal multiple stationary points. In addition, SPO problems are multiobjective in nature. Traditional optimization techniques have the problem of only one criterion being optimized at a time ([3], [10]).

The drawbacks of classical optimization techniques have led to the growth of research in the field of evolutionary computing (EC). EC techniques do not require explicit knowledge of the problem structure or differentiability, and have the ability to provide multiple near-optimal solutions to even ill-defined problems. In the field of SPO, a number of authors ([5], [15]) are now adopting GAs with embedded FE solvers to automate the search for good quality solutions. These approaches have been shown to offer a more promising approach to SPO problems, and are emerging as alternative optimization strategies for solving these problems. They are proving to be robust in delivering global optimal quality solutions and are helping to resolve some of the complexity issues encountered in SPO problems [12]. They also offer the benefit of cataloging the optimal solutions for future reuse. This can save design time and effort for future problems. Roy et al. [14] implemented an adaptive micro-GA for shape optimization of process variables in multipass wire drawing processes. Hwang and Chung [6] proposed a modified micro-GA for the optimization of die shape in extrusion. These GA-based techniques provide an algorithmic framework to deal with SPO problems. These techniques can deliver multiple good solutions, which speeds up the design process. However, the GA-based approach using the FE solver as an embedded optimizer incurs severe computational cost in real-life problems. This is because the objective functions are often analytically unknown, and hence function evaluations can only be achieved through costly computer simulations. Therefore, the majority of research effort in these areas has focused on the development of computationally efficient algorithms ([2], [8]). However, very little research effort has been devoted to handling the fundamental issue in SPO problems, namely, the interstage dependency link. Since the design of a rolling system is characterized by its multistaged sequential nature, this paper proposes an optimization approach for handling this challenge using GAs.

\section{Proposed Optimization ApPROACH}

This section proposes an optimization approach for handling the sequential nature of real-life process optimization problems. The solution strategy adopted by the optimization approach is based on the GA. The dependency link between stages is modeled by informing the subsequent stage $(j)$ of the move made by the $(j-1)$ th stage such that the solution alternatives considered for the $j$ th stage take into account the move made by the $(j-1)$ th stage. The solution strategy is only applicable to strictly sequential processes. For example, in roll design, we model "passes" 


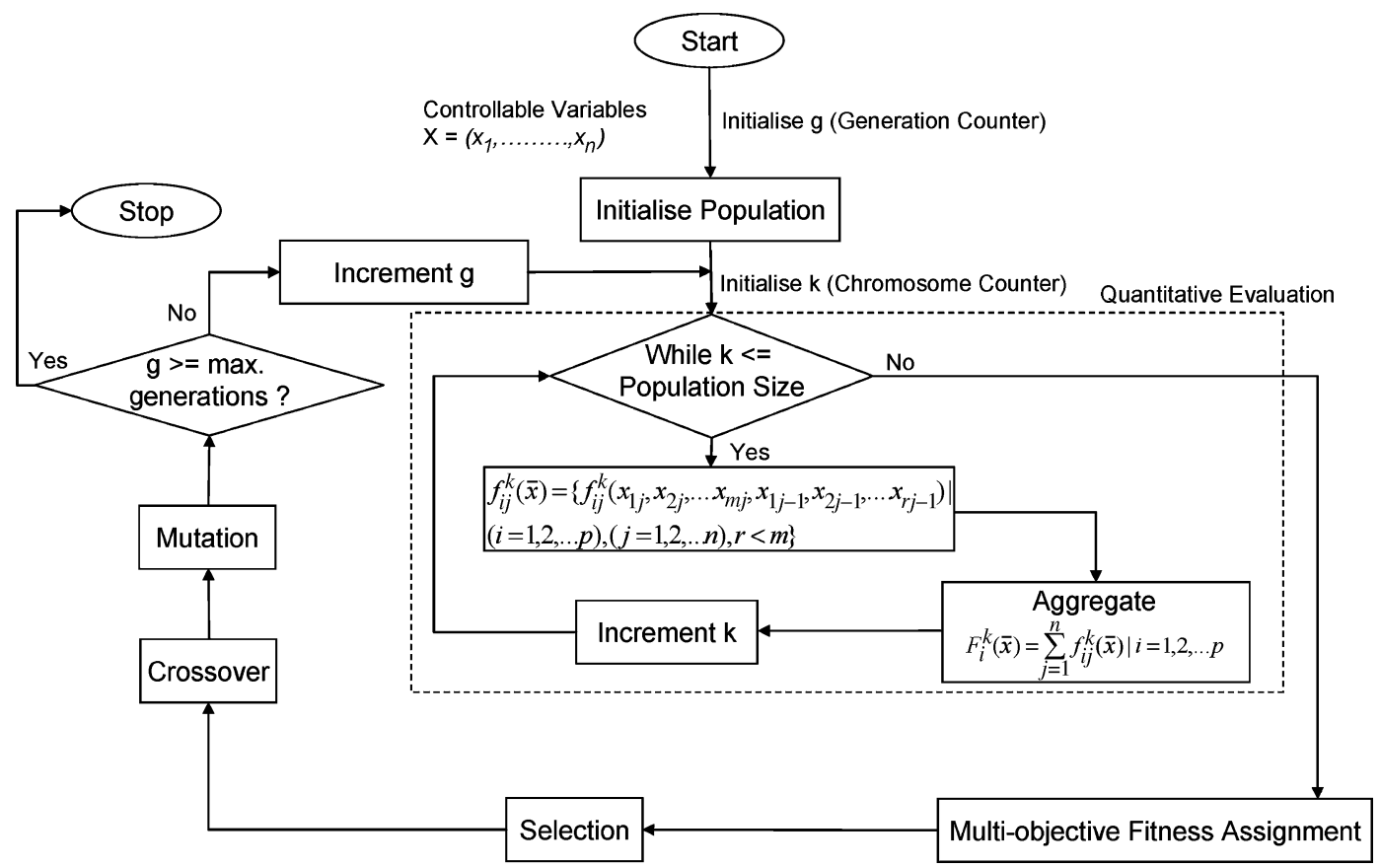

Fig. 4. NSGA-II-based proposed optimization approach for SPO.

\begin{tabular}{|c|c|c|c|c|c|c|c|c|}
\hline$x_{11}$ & $x_{21}$ & $x_{m 1}$ & $x_{12}$ & $x_{22}$ & $x_{m 2}$ & $x_{1 n}$ & $x_{2 n}$ & $x_{m n}$ \\
\hline 1000. & $1011 \ldots$ & 1110. & 1000. & 1011.. & 0010. & 1001.. & $0001 \ldots$ & 0110. \\
\hline
\end{tabular}

Fig. 5. String structure of a chromosome.

as "stages," whereby the output stock of one-pass/stage serves as an input into the deforming tool of the other pass/stage. This example is illustrated in Fig. 3.

The GA-based approach was selected because GAs have been used extensively as search and optimization tools in various problem domains and the primary reasons for their success are applicability, ease of use, and global perspective. NSGA-II was selected being the most robust and popular multiobjective optimization algorithm [3]. The solution strategy is coded in $\mathrm{C}++$ using the proposed algorithm shown in Fig. 4. The solution algorithm consists of three main parts: the NSGA-II algorithm, the multistage model, and the multiobjective fitness assignment. The initial population is created by giving random values to the decision/controllable variables of the problem. The termination criterion of the solution algorithm is purely based on the number of generations specified by the user. Since the solution algorithm uses NSGA-II as its optimization engine, its computational complexity is $\mathrm{O}\left(\mathrm{MN}^{2}\right)$ (where $\mathrm{M}$ is the number of objectives and $\mathrm{N}$ is the population size), which is the same as that of NSGA-II.

Individual members of the population are evaluated in two steps. The first step is a local evaluation of a subset of strings in the chromosomes. This represents the objective function values for each stage of the SPO problem. The second part is a global evaluation that aggregates the objective function values of all the stages by using a suitable aggregation operator. The objective function value of the global evaluation determines the fitness of the chromosome. The dependency relationship is modeled by incorporating design variables from a previous pass $j-1$ into the objective function of pass $j$. This is peculiar to the sequential processes in which the output stock from one stage serves as input stock to the other stage.

The multiobjective fitness assignment, selection and reproduction follow NSGA-II [4]. The population is sorted based on nondomination. Each solution is assigned a fitness equal to its nondomination level ( 1 is the best level). Thus, minimization of fitness is assumed. NSGA-II uses an elitist approach through a selection operator that creates a mating pool by combining the parent and child populations. This elitism ensures that the "good" solutions of the population are not lost, thereby creating a selection pressure towards the global Pareto front. The crowded tournament selection operator is used to select new offspring. That is, between two solutions with different nondomination ranks, the solution with the lower rank is preferred. Otherwise, if both the solutions belong to the same front, then the solution that is located in a region with lesser number of solutions is preferred. In this way, the operator guides the selection process at various stages of the algorithm towards a uniformly spread Pareto front. Crossover and mutation operators are used to create a child population. Due to the large number of variables in SPO problems, a multipoint crossover operator is used to ensure a more effective transfer of genetic material during reproduction.

Fig. 5 illustrates the string structure adopted by the proposed approach. A genetic string is made up of substrings representing the number of stages, where the number of stages is assumed to be fixed. Since there is only one finishing stage, the genetic string has $n-1$ string segments for roughing and one segment 
TABLE I

Design Details of Four-Stage Rolling Problem

\begin{tabular}{c|c|c|c}
\hline Pass No & Design Variables & Bits & Design Variable Bounds \\
\hline \multirow{3}{*}{1} & Semi-height $\left(h_{1}\right) ;$ Semi-width $\left(w_{1}\right) ;$ & $9 ; 10 ;$ & $20<=h_{1}<=25 ; 60<=w_{1}<=75 ;$ \\
& Roll radius $(R r) ;$ Roll Gap $(R g) ;$ & $12 ; 7 ;$ & $300<=R r<=350 ; 4<=R g<=6 ;$ \\
& Temperature $(T) ;$ Roll Speed $(N)$ & $14 ; 10$ & $900<=T<=1200 ; 25<=N<=40$ \\
\hline \multirow{3}{*}{2} & Pass Diameter $(D) ;$ Roll radius $(R r) ;$ & $8 ; 10 ;$ & $28<=D<=32 ; 295<=R r<=315 ;$ \\
& Roll Gap $(R g) ;$ Temperature $(T) ;$ & $7 ; 14 ;$ & $4<=R g<=6 ; 900<=T<=1150 ;$ \\
& Roll Speed $(N)$ & 10 & $35<=N<=50$ \\
\hline \multirow{3}{*}{3} & Semi-height $\left(h_{1}\right) ;$ Semi-width $\left(w_{1}\right) ;$ & $9 ; 9 ;$ & $12<=h_{1}<=18 ; 40<=w_{1}<=50 ;$ \\
& Roll radius $(R r) ;$ Roll Gap $(R g) ;$ & $12 ; 7 ;$ & $250<=R r<=300 ; 4<=R g<=6 ;$ \\
& Temperature $(T) ;$ Roll Speed $(N)$ & $14 ; 11$ & $900<=T<=1125 ; 65<=N<=90$ \\
\hline \multirow{3}{*}{4} & Pass Diameter $(D) ;$ Roll radius $(R r) ;$ & $8 ; 12 ;$ & $18<=D<=22 ; 250<=R r<=300 ;$ \\
& Roll Gap $(R g) ;$ Temperature $(T) ;$ & $7 ; 14 ;$ & $4<=R g<=6 ; 800<=T<=1100 ;$ \\
& Roll Speed $(N)$ & 11 & $80<=N<=120$ \\
\hline
\end{tabular}

for the finishing pass. Each substring consists of product and process variables. Only controllable variables are represented in the genetic string.

\section{EXPERIMENTAL DETAILS AND DisCUSSION OF RESUlTS}

Results were obtained using the multipass RSD mathematical model developed in Section II-B. The proposed NSGA-IIbased optimization approach was applied to the mathematical model to minimize deformation load while maximizing mill throughput. Experiments were carried out using the proposed approach for the two objectives and four passes to illustrate how the algorithm deals with a multiobjective, multipass search space. The following additional parameters were set for the experiment: $P_{\max }=750 \mathrm{kN}$ per stand, final rod diameter $(D)=$ $20.1 \pm 0.25 \mathrm{~mm}$, where $k_{\min }$ is set as $19.85 \mathrm{~mm}$ and $k_{\max }$ is set as $20.35 \mathrm{~mm}$, and the tolerance range was estimated based on design experience.

\section{A. Experimental Details}

Each variable is encoded first, and then linked together as a chain to form the chromosome. Design variables encoded for the rolling problem are semi-height $\left(h_{1}\right)$, semi-width $\left(w_{1}\right)$, roll radius $(R r)$, roll gap $(R g)$, temperature $(T)$, and roll speed $(N)$. The variable bounds are estimated using feasible design values from literature [18]. This is stated as follows for pass 1 : $20<=h_{1}<=25 ; 60<=w_{1}<=75 ; 300<=R r<=350$; $4<=R g<=6 ; 900<=T<=1200 ; 25<=N<=$ 40. The granularity for the design variables is chosen to be 0.01 . The number of bits representing a design variable is $a$ if $2^{a-1}<=$ (upper limit - lower limit)/granularity $<=2^{a}$. This implies that the number of bits representing $h_{1}$ is 9 since $2^{8}<=(25-20) / 0.01<=2^{9}$; similarly, 10 bits represent $w_{1}, 12$ bits represent $R r, 7$ bits represent $R g, 14$ bits represent $T$, and 10 bits represent $N$. The bits representing each design variable are finally concatenated as a binary chain. The total resulting bit number of the chromosome is 225 . Details of the variable encoding are shown in Table I.

The performance of the solution approach was investigated for different values of crossover and mutation probabilities. Ten independent GA runs were performed in each case using a different random initial population. The crowded tournament selection operator [4] was used to select new offspring. The best results were obtained with the following parameters: population of size 500 for 2000 generations with a three-point crossover probability of 0.8 and a mutation probability of 0.03 , and a tournament selection of size 3 . The crossover points in the chromosomes were randomly selected. Due to the large chromosome length of 225 bits, the three-point crossover was chosen to enable more effective transfer of genetic material during reproduction than the standard two-point crossover. Six out of ten runs with different random number seeds obtained similar results. The results reported in Section V-B were obtained from one of the six runs that produced similar results. The remaining four runs showed similar convergence but inferior diversity of solutions. The computational complexity of the algorithm is $\mathrm{O}\left(2 * 500^{2}\right)$ (where 2 is the number of objectives and 500 is the population size). Using a $2.80 \mathrm{GHz}$ computer with a Pentium 4 Processor, each run took about 28 minutes. The solutions were evaluated using direct calculations rather than numerical simulations. A random search of 10000 points was also conducted in order to get an indication of the search space. This was used to identify the likely presence of a Pareto front in the design problem.

\section{B. Experimental Results and Discussion of Results}

This is a multiobjective, sequential, multistaged, constraint process optimization problem, with interstage dependency links. Fig. 6 illustrates the global search space of the problem in objective function space. The following observations can be made from this figure regarding the search space of this problem.

- The existence of a Pareto front can be seen from Fig. 6. This implies that there is conflict between the two objectives. By fitting a curve to the optimized solutions, it can be seen that the Pareto front is nonlinear, and a combination of convex and concave regions.

- There is a bias in the search space towards a region that corresponds to the $T_{p}$ and $P_{T}$ values lying between 400 and $600 \mathrm{~mm}^{3} / \mathrm{s}$ and between 1000 and $1400 \mathrm{kN}$, respectively.

- The boundary of the randomly generated 10000 solutions does not coincide with the near-optimal Pareto front identified by the set of solutions obtained by the algorithm. This situation arises because the overall objective function is a summation of the objective functions for individual stages. Identification of Pareto optimal solutions requires that the information about the previous stage is taken into account 


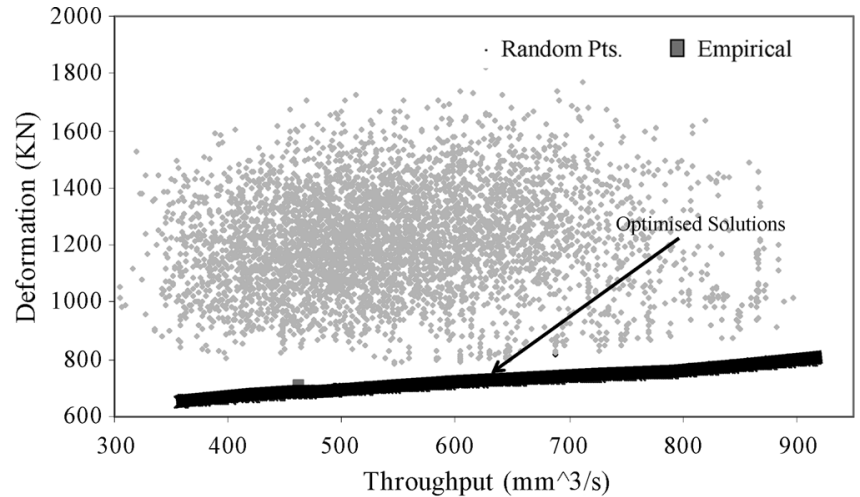

(a)

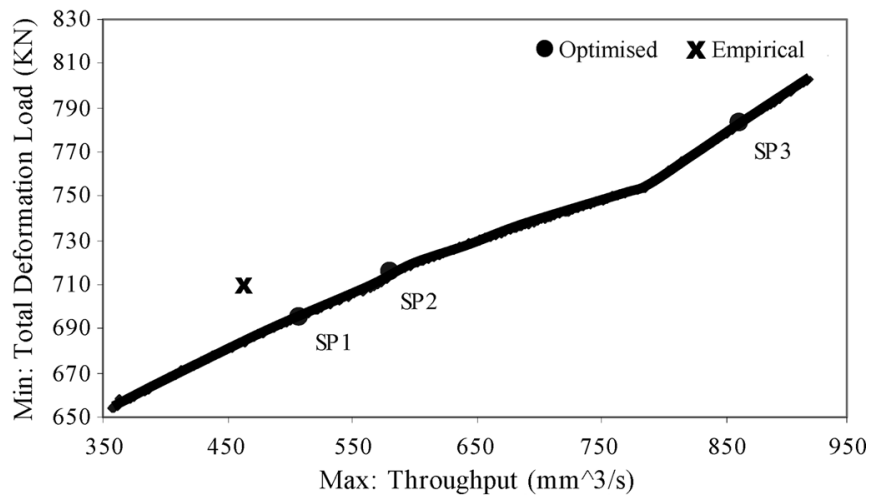

(b)

Fig. 6. Pareto front for four pass rolling process. (a) Complete picture of the search space. (b) Blown-up view of a part of the search space.

by the next stage. There is a low density of population in the region between the boundary of random solutions and the near-optimal Pareto front. This is because there are very few solutions existing in this region of the search space.

- The local search spaces corresponding to the individual stages are uniform and constrained, with continuous Pareto fronts.

The results obtained from the proposed optimization approach are compared with empirical results published in the literature [18]. The optimized results (after 2000 generations) shown in Fig. 6 were obtained for the parameters outlined in Section V-A. Comparison of the random search space and the results achieved from the algorithm confirms that the solution algorithm has been able to converge to the Pareto front. The solid line shown in Fig. 6(a) is composed of several optimized solutions "x." Since the search space is not known in absolute terms, the results reported in Fig. 6 have converged to the near-optimal Pareto front locating a reasonable spread of multiple optimized solutions. The presence of a Pareto front also confirms the conflicting relationship between deformation load and throughput. The empirical design point obtained from the literature was superimposed on the search space and compared with the near-optimal solutions. Since this point does not lie on the identified near-optimal Pareto front, it is clear that the solutions obtained are superior to the empirical solution.

Out of the optimized solutions identified by the proposed optimization approach, three are randomly selected [SP1, SP2, and

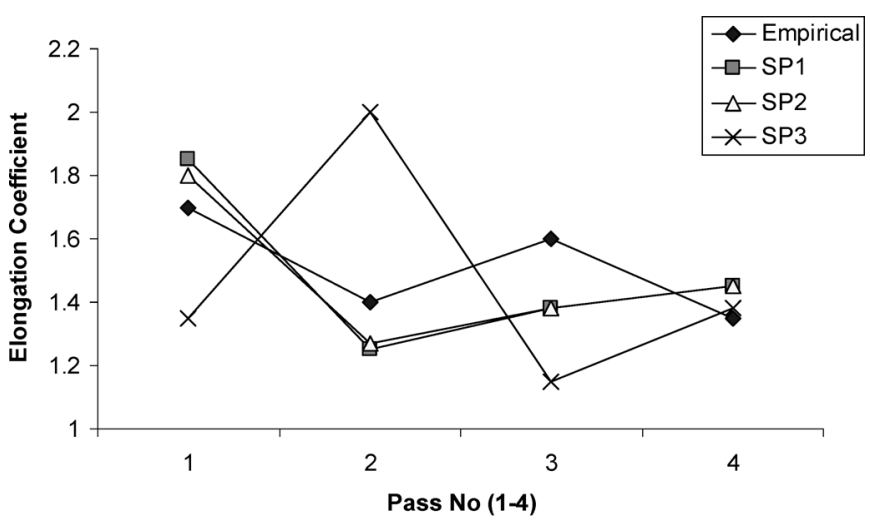

(a)

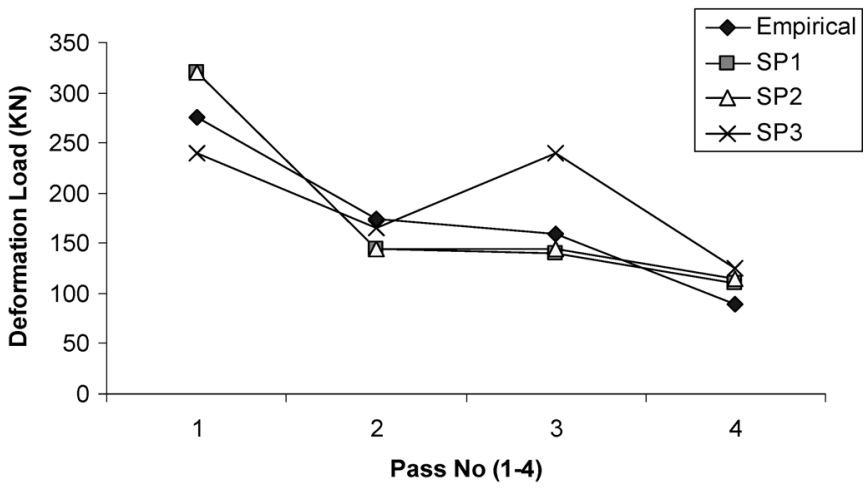

(b)

Fig. 7. Selected Pareto solutions for four-pass rolling process. (a) Elongation coefficient. (b) Deformation load.

SP3-located in Fig. 6(b)] and compared with the empirical solution in terms of throughput, deformation load, elongation coefficient, and the deformed sections. These solutions are shown in Fig. 7 and their nature is outlined in Table II. Table II identifies the near-optimal breakdown sequence of the stock deformation from the proposed optimization approach.

Results obtained from the proposed approach also provide insight into the complex behavior of the design problem. Fig. 7 shows the complex relationship between elongation coefficient and the deformation load. The deformation load behavior shows a similar downward trend for the four passes when compared with the empirical solution. Although the deformation load for point 3 at pass 3 appears to show an upward trend at $243 \mathrm{kN}$, this is due to the large input stock area entering the roll at pass 3 from the previous deformation. It is expected that large cross sections would incur high deformation load and hence high elongation coefficient values, since the load deforms the stock in the direction of rolling. However, point 3 at pass 3 shows a conflict. The result shows a high load deformation, low elongation coefficient for a large section. This can be attributed to the large coefficient of spread of SP1 and SP2 at pass 3 (1.36) compared with that of SP3 at pass 3 (1.17). Coefficient of spread is defined as the percentage increase in breadth of the bar during rolling. The behavior of the spread coefficient correlates with load deformation. A large spread coefficient results in large deformation load and vice versa. This is intuitive since a large spread coefficient implies higher contact area, which results in a higher deformation load. 
TABLE II

Design Details of Four-Stage Rolling Problem

\begin{tabular}{c|c|c|c|c}
\hline Rolling Parameters & Empirical Schedule & SP1 & SP2 & SP3 \\
\hline Input Stock & Square & $41 \times 41$ & $41 \times 41$ & $41 \times 41$ \\
1 & Oval 21.1 67.5 & $18.1 \times 75$ & $18.4 \times 75$ & $25 \times 75$ \\
2 & Round Ø29.5 & $\varnothing 30.2$ & $\varnothing 30.2$ & $\varnothing 28$ \\
3 & Oval 14.8 4 43 & $15.7 \times 50$ & $15.7 \times 50$ & $15.7 \times 50$ \\
4 & Round Ø20.1 & $\emptyset 20.19$ & $\emptyset 20.22$ & $\emptyset 20.11$ \\
\hline
\end{tabular}

\section{DISCUSSION AND CONCLUSION}

This paper describes the optimization of a real-life multipass RSD problem using an approach based on GAs. The design of a rolling system is a multiobjective, multistage SPO problem involving a large number of design variables and a sequential relationship between any two stages. The majority of recent research effort in this area has focused on the development of computationally efficient algorithms. However, very limited research effort has been devoted to handling the fundamental issue in these problems, namely, the interstage dependency link.

To address this gap in current research, a GA-based optimization approach is applied to handle the dependency relationship between multiple stages in these problems. Validation of the results is performed based on the visualization of the search space in the presence of a random search, published results, and the nearoptimal solutions from the proposed optimization approach. Visual comparison of the results of the random search and the results achieved from the proposed optimization approach confirms that the latter has been able to converge to the near Pareto front with a good spread of solutions. Performance comparison with the result reported in the literature confirms that the near-optimal solutions obtained by the proposed optimization approach dominate the result reported in the literature. The near-optimal solutions obtained from the proposed algorithm offer the capability to designers to tradeoff solutions at various dimensions such as parameter level, objective level and interstage level. The proposed optimization approach is, however, limited to process optimization problems with sequential relationships between consecutive stages, and does not consider interstage dominations.

There are a number of proposed future research activities for solving SPO problems within a multistage optimization framework. It would be useful to investigate the nature of interactions between the different stages during the evolutionary search. Since the problem parameters are real, it would also be interesting to test the performance of the algorithm with real vector representation. Finally, further study could consider developing techniques for addressing the interstage dominations in process optimization problems.

\section{REFERENCES}

[1] J. Bard, Practical Bilevel Optimization Algorithm and Applications. Norwell, MA: Kluwer, 1998.

[2] R. Brekelmans, L. Driessen, H. Hamers, and D. D. Hertog, "Constrained optimization involving expensive function evaluations: A sequential approach," Eur. J. Oper. Res., vol. 160, pp. 121-138, 2005.

[3] K. Deb, Multi-Objective Optimization Using Evolutionary Algorithms. New York: Wiley, 2001.

[4] K. Deb, A. Pratap, S. Agarwal, and T. Meyarivan, "A fast and elitist multi-objective genetic algorithm: NSGA-II," Indian Inst. Technol. (IIT), Kanpur, India, KanGAL Rep. 200002, 2000.

[5] B. Filipic and B. Sarler, "Evolving parameter settings for continuous casting of steel," in Proc. 6th Eur. Congr. Intell. Tech. Soft Comput., Aachen, Germany, 1998, Verlag Mainz.
[6] S. M. Hwang and J. S. Chung, "Application of genetic algorithm to optimal design of the die shape in extrusion," J. Mat. Process. Technol., vol. 72, pp. 69-77, 1997.

[7] M. S. Joun and S. M. Hwang, "Optimal process design in steady-state metal forming by finite element method-II," Int. J. Mach. Tool Manuf., vol. 33, no. 1, pp. 63-70, 1993.

[8] A. C. Keys and L. P. Rees, "A sequential-design metamodeling strategy for simulation optimization," Comput. Oper. Res., vol. 31, pp. 1911-1932, 2004.

[9] S. Kobayashi, "Process design in metal forming by the finite element method," Advanced Technol. Plasticity, vol. 11, pp. 1213-1219, 1987.

[10] S.-E. Lundberg, "Roll pass design: The key function in control of shape, dimension and mechanical properties of hot rolled products," Scandinavian J. Metallurgy, vol. 26, no. 3, pp. 102-114, 1997.

[11] V. Oduguwa, "Rolling system design optimization using soft computing techniques," EngD. thesis, School of Industrial and Manufacturing Science, Cranfield Univ., Cranfield, U.K., 2003.

[12] V. Oduguwa, A. Tiwari, and R. Roy, "Evolutionary computing in manufacturing industry: An overview of recent applications," Appl. Soft Comput., vol. 5, pp. 281-299, 2005.

[13] K. Osakada, G. B. Yang, T. Nakamura, and K. Mori, "Expert system for cold-forging process based on FEM simulation," Ann. CIRP, vol. 39, no. 1, pp. 249-252, 1990.

[14] S. Roy, S. Ghosh, and R. Shivpuri, "Optimal design of process variables in multi-pass wire drawing by genetic algorithms," J. Manuf. Sci. Eng., pp. 118-124, 1996.

[15] B. Sarler, B. Filipic, M. Raudensky, and J. Horsky, "An interdisciplinary approach towards optimal continuous casting of steel," in Proc. TMS, Nashville, TN, 2000, pp. 27-36.

[16] T. Shinokura and K. Takai, "A new method for calculating spread in rod rolling," J. Appl. Metalworking, vol. 2, pp. 147-160, 1982.

[17] H. P. Williams, Model Building in Mathematical Programming. New York: Wiley, 1999.

[18] Z. Wusatowski, Fundamentals of Rolling. New York: Pergamon, 1969.

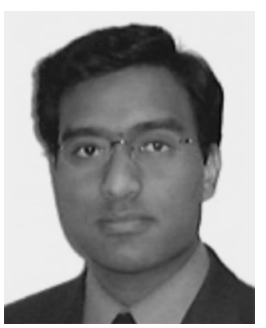

Ashutosh Tiwari is leading the research in applied soft computing within the Decision Engineering Centre, Cranfield University, Bedfordshire, U.K., and has developed new research areas in the application of soft computing to product and process design. He is an Editorial Board Member of the Applied Soft Computing Journal from Elsevier and an Associate Editor of the International Journal of Applied Fuzzy Sets Theory from Research Sciences Press. He has published extensively in his research career: over 58 research papers in refereed journals, conferences and books, and an edited book on applied soft computing.

Victor Oduguwa is the Head of Design and Value Engineering, Mobile Telecommunications Network, Nigeria. He has participated as organizer of major international conferences and has published applied soft computing papers in journals and conferences over the last five years. His research interest includes application of soft computing for real-life engineering design optimization.

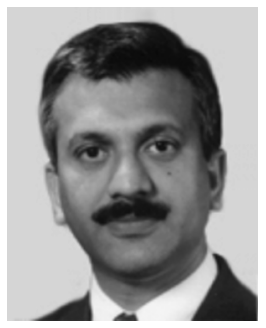

Rajkumar Roy is the Head of the Decision Engineering Centre, Cranfield University, Bedfordshire, U.K. He is the Editor-in-Chief of the Applied Soft Computing Journal from Elsevier and a Book Series on Decision Engineering (Springer). He has published applied soft computing papers in Journals and Conferences over the last 11 years. His research interest includes application of soft computing for real life design optimization.

Prof. Roy is also the Chairman of the World Federation of Soft Computing (WFSC), 